\title{
Idiopathic Membranous Nephropathy with Solitary Immunoglobulin A Deposition: A Case Report and a Review of the Literature
}

\author{
Bin $\mathrm{Li}^{1-3}$, Hailiu Huang ${ }^{4}$, Shicong Yang ${ }^{5}$, Xin Wang ${ }^{1-3}$, Wei Chen ${ }^{1-3}$, \\ Zhijian $\mathrm{Li}^{1-3}$ and Xionghui Chen ${ }^{1-3}$
}

\begin{abstract}
:
A 63-year-old man with an 8-year history of proteinuria was diagnosed with nephrotic syndrome, and a renal biopsy was performed. Light and electron microscopic analyses showed classic features of idiopathic membranous nephropathy (IMN). However, immunofluorescence tests revealed solitary polyclonal granular IgA deposition along the glomerular capillary walls, rather than IgG, which is often dominant in IMN. The combined use of corticosteroids and calcineurin inhibitor was noticeably effective in reducing proteinuria and improving edema in the current case. Two additional rare cases of IMN with solitary IgA deposition were reviewed, and long-term surveillance is still warranted to characterize its clinicopathological features and outcome.
\end{abstract}

Key words: membranous nephropathy, solitary polyclonal IgA deposition, rare disease, case report

(Intern Med Advance Publication)

(DOI: 10.2169/internalmedicine.8404-21)

\section{Introduction}

Membranous nephropathy (MN) is one of the most common causes of nephrotic syndrome (NS) in adults worldwide. It is characterized by the presence of diffuse thickening of the glomerular capillary wall on light microscopy as a result of an immune complex deposition on the extracapillary side of the glomerular basement membrane (GBM). The immune deposits contain immunoglobulin, antigen and complement molecules when imaged with immunofluorescence and present as subepithelial electron-dense deposits (EDDs) under electron microscopy. Approximately $75 \%$ of $\mathrm{MN}$ cases are idiopathic (idiopathic membranous nephropathy or IMN), and the remaining $25 \%$ are associated with various autoimmune, infectious or malignant diseases (secondary membranous nephropathy or SMN) (1). Immunosuppressive agents are central to the treatment of $\mathrm{MN}$, but the clinical course of MN is unclear and variable, ranging from sponta- neous remission in $30 \%$ of patients to progressing toward end-stage renal disease within 5-15 years in $40 \%$ of patients (2).

The pathological findings of IMN are characterized by predominant immunoglobulin $\mathrm{G}(\mathrm{IgG})$ and/or complement 3 (C3) deposition. Accumulating immunohistological findings have proposed that IgG4 is the premier IgG subclass in the glomerular immune deposits of IMN (3). Consistently, recent advances have shown that anti-podocyte antibodies against the two mostly well-recognized target antigens in IMN (phospholipase A2 receptor (PLA2R) and thrombospondin type-1 domain-containing 7A) are present in 70\%$80 \%$ of IgG4 subtype cases (1). By contrast, in SMN, the deposits are frequently not IgG4-dominant, and the codeposition of $\operatorname{IgA}$ with $\operatorname{IgG}$ is only observed in $5 \%$ of $\mathrm{MN}$ patients $(3,4)$. Malignancy-associated SMN features the absence of $\operatorname{IgG} 4$ and the presence of $\operatorname{IgG} 1$ and $\mathrm{IgG} 2$ in renal biopsies (5), while in SMN such as membranous lupus nephritis (LN), IgG and $\mathrm{C} 3$ deposits are usually accompanied

\footnotetext{
${ }^{1}$ Department of Nephrology, The First Affiliated Hospital, Sun Yat-sen University, China, ${ }^{2}$ Key Laboratory of Nephrology, Ministry of Health, China, ${ }^{3}$ Guangdong Provincial Key Laboratory of Nephrology, China, ${ }^{4}$ Department of Nephrology, The Second People's Hospital of Zhaoqing, China and ${ }^{5}$ Department of Pathology, The First Affiliated Hospital, Sun Yat-sen University, China 


\section{by $\operatorname{IgA}$ and/or C1q (6).}

Each subclass of immunoglobulin has unique biological activities, and these subclasses may be preferentially produced in response to different antigens. However, the involvement of different immunoglobulin subclasses in the pathogenesis of $\mathrm{MN}$ is still not fully elucidated. To our knowledge, only two unusual IMN cases with solitary polyclonal IgA deposition have been reported by Japanese authors (2015 and 2019) (7, 8). Findings from these two cases are consistent with regard to the main features of granular deposition of solitary polyclonal $\operatorname{IgA}$ on immunofluorescence and quick remission upon the administration of immunosuppressive induction therapy. We herein report an additional case of IMN with solitary $\operatorname{IgA}$ deposition and clarify the characteristics of this unique entity through a literature review.

\section{Case Report}

A 60-year-old man with an 8-year history of proteinuria $(1+$ to $3+)$ and hematuria $(1+$ to $3+)$ was referred to our hospital due to generalized edema of the lower extremities and foamy urine. One year ago, he had been diagnosed with acute myocardial infarction and underwent coronary stent implantation; thereafter, he was treated with aspirin and clopidogrel. On admission, a physical examination showed pitting edema in his lower extremities; however, no abnormal signs were observed in the lungs, heart or abdomen. A urinalysis revealed proteinuria $(3+; 3.76 \mathrm{~g}$ daily) and hematuria (2+; red blood cell count: 15/high-power field). Laboratory studies revealed a hemoglobin level of $13.9 \mathrm{~g} / \mathrm{dL}$, and serum chemistry showed a blood urea nitrogen level of 38.4 $\mathrm{mg} / \mathrm{dL}$ and serum creatinine level of $0.62 \mathrm{mg} / \mathrm{dL}$, low total serum protein level of $4.1 \mathrm{~g} / \mathrm{dL}$, albumin level of $2 \mathrm{~g} / \mathrm{dL}$ and total cholesterol level of $162.79 \mathrm{mg} / \mathrm{dL}$, indicating a diagnosis of NS. In addition, immunological tests showed a reduced serum IgG level (728 mg/dL), slightly increased serum C3 level $(127 \mathrm{mg} / \mathrm{dL})$ and normal levels of serum IgA, IgM and C4 (272, 128 and $16 \mathrm{mg} / \mathrm{dL}$, respectively), and the results of other serological tests were negative for antinuclear antibodies, anti-double-stranded DNA antibodies, anti-Smith antibodies, anti-glomerular basement membrane (GBM) antibodies, anti-neutrophil cytoplasmic antibody and cryoglobulin. All viral serological markers were negative. Serum tumor markers, including alpha-fetoprotein, carcinoembryonic antigen and carbohydrate antigen 19-9, were all negative, as were serologic tests for hepatitis B surface antigens and anti-hepatitis $\mathrm{C}$ antibodies. No serological or urinary Bence-Jones proteins were detected. The most common and frequent infectious diseases, including skin infection, sinusitis, bronchitis, pneumonia, gastrointestinal infection and chronic diarrhea, were not observed. However, abdominal ultrasonography revealed that both kidneys had a normal size and echogenicity. As described above, none of the examinations suggested a distinct secondary cause of NS.
We subsequently obtained the patient's informed consent to perform a percutaneous renal biopsy. Light microscopy showed sclerosing lesions in 1 of 11 glomeruli, and diffuse thickening of the GBM was observed in all glomeruli. Masson staining showed granular eosinophilic immune complex deposition along the subepithelial area of the GBM. Periodic Schiff-Methenamine staining showed global thickening of glomerular capillary walls, with the absence of spike-like formation (Fig. 1A, B). In accordance with the above histological alterations that were indicative of IMN, electron microscopic examinations also supported the diagnosis of IMN with a slightly thickened GBM, global subepithelial EDDs and extensive foot process effacement of the podocyte (Fig. 2A, B).

However, immunofluorescence microscopy showed strong IgA- (Fig. 1D), IgA1- (Fig. 1E) and IgA2- (Fig. 1F) dominant deposition along the GBM, with weak positivity of $\mathrm{C} 1$ q, $\kappa-$ (Fig. 1G) and $\lambda$ - (Fig. 1H) light chains and negative staining for anti-IgG (Fig. 1C), IgG1, IgG2, IgG3, IgG4, IgM, C3, Fg and PLA2R. Direct immunofluorescence tests of $\operatorname{IgA}$ and $\operatorname{IgG}$ were performed more than three times. However, no subendothelial EDDs, punctate powdery formed deposits, organized deposits or amyloid fibrils were detected. Based on the above-mentioned pathological findings, clinical manifestations and exclusion of other secondary factors, a diagnosis of IMN with solitary IgA deposition was made.

During the first admission, given the low-grade proteinuria $(<4 \mathrm{~g} /$ day) and normal renal function in the present case (9), neither corticosteroid nor immunosuppressive therapy was started aside from supportive care, which consisted of the use of an angiotensin II receptor blocker (valsartan), lipid-lowering agents (atorvastatin and ezetimibe) and antiplatelet drug (clopidogrel). However, no remission was observed 4 months later at the second admission, and the 24-h urine protein continuously increased to $4.76 \mathrm{~g}$, while serum albumin decreased to $1.8 \mathrm{~g} / \mathrm{dL}$, accompanied by worsened swelling of the extremities, eyelid edema and oliguria. The combined use of steroid and calcineurin inhibitor (prednisolone $30 \mathrm{mg}$ daily for 8 weeks, tacrolimus $3 \mathrm{mg}$ every $12 \mathrm{~h}$ for 1 week with a target blood concentration of $3-5 \mathrm{ng} / \mathrm{mL}$ and then gradually tapered) was administrated (9).

After two months of treatment, the present case was completely resolved, as evidenced by the disappearance of proteinuria and hematuria on a urinalysis. The proteinuria declined to $<0.15 \mathrm{~g}$ per day, and the serum albumin normalized to $3.9 \mathrm{~g} / \mathrm{dL}$ without symptoms of edema. The patient has been closely followed thus far, but exhaustive screening tests to determine the underlying cause of SMN has yet to yield any clues.

\section{Discussion}

We encountered a pathologically diagnosed IMN patient with typical membranous features on light and electron microscopic analyses of renal biopsy tissue. Of note, granular 

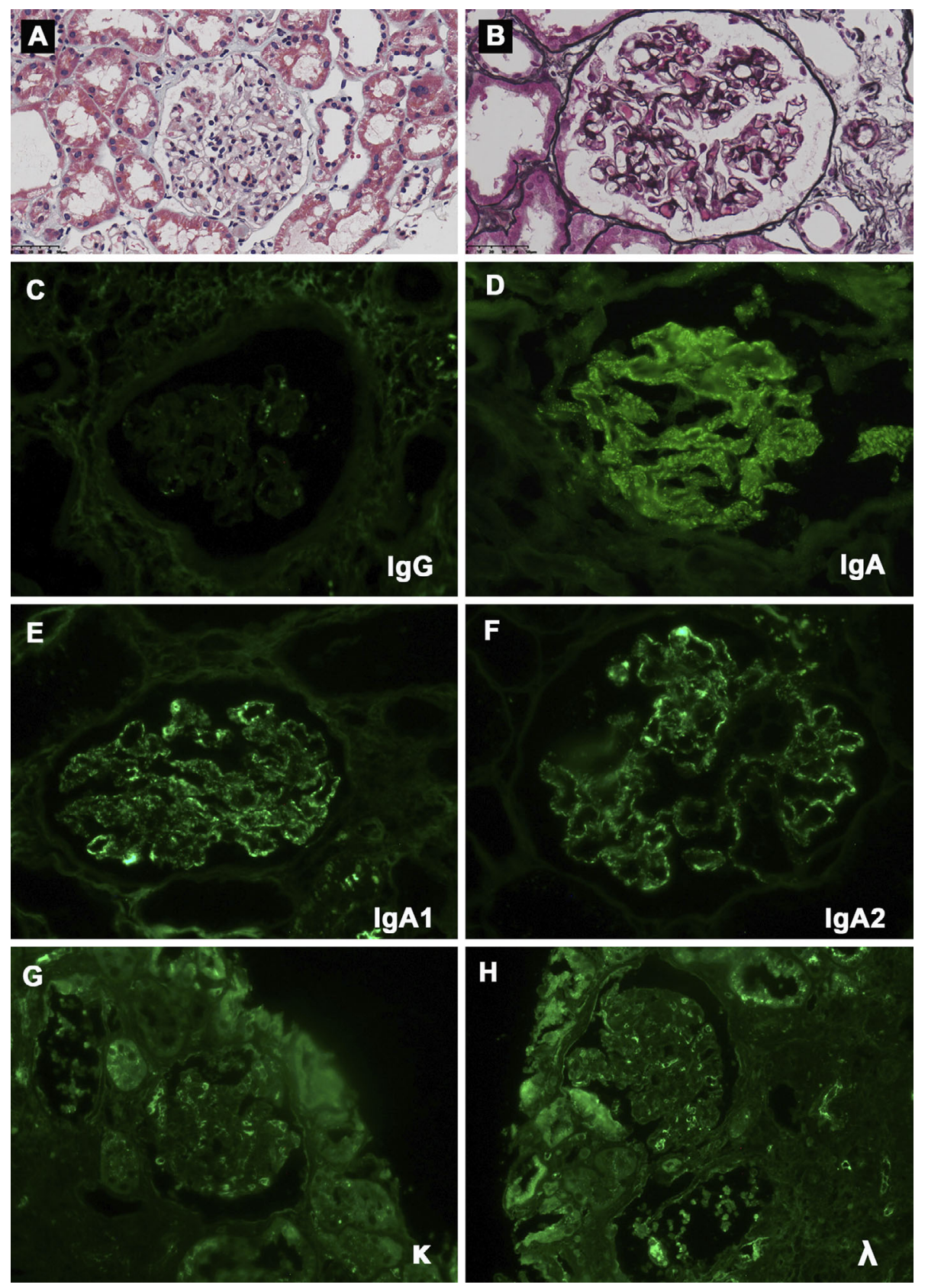

Figure 1. Light microscopy findings and immunofluorescent staining. a and b: Masson and Periodic acid-Methenamine staining of the glomeruli showing diffuse thickening of the GBM and granular eosinophilic immune complex deposition in the subepithelial area (original magnification $\times 400$ ). Granular staining of IgG (c), IgA (d), IgA1 (e), IgA2 (f), $\kappa$ light chain (g) and $\lambda$ light chain (h) along the glomerular capillary walls is shown (original magnification $\times 400)$.

deposition of solitary $\operatorname{IgA}$, but not $\operatorname{IgG}$, $\operatorname{IgM}$ or C3, was observed along the glomerular capillary walls on immunofluorescence; however, no underlying secondary diseases were found during the follow-up period. Therefore, the clinical and pathological findings, except for those of immunofluorescence, were all compatible with a diagnosis of IMN. To our knowledge, this is the third reported case of IMN with solitary IgA deposition.

Mounting evidence indicates that the distinction between IMN and SMN can be suggested by IgG subclasses. An already-known fact is that most renal biopsies with IMN are characterized by IgG4-dominant glomerular deposits (10). In contrast, the presence of $\operatorname{IgG} 1, \operatorname{IgG} 2, \operatorname{IgG} 3, \operatorname{IgM}, \operatorname{IgA}$, and $\mathrm{C} 1 \mathrm{q}$ is closely related to $\operatorname{SMN}(5,10)$. In particular, secondary causes should be suspected when IgG deposits are accompanied by IgA deposits (4). Therefore, in our current case, although the depositions of IgG and all IgG subclasses were deficient, the markedly elevated $\operatorname{IgA}$ depositions were highly suggestive of SMN at the outset, and various attempts were made to determine the cause of the putative SMN; however, despite two years of such efforts, the results of all examinations excluded the possible existence of autoimmune disease, infection and malignancy. For example, $\mathrm{LN}$, the most common visceral manifestation of systemic lu- 


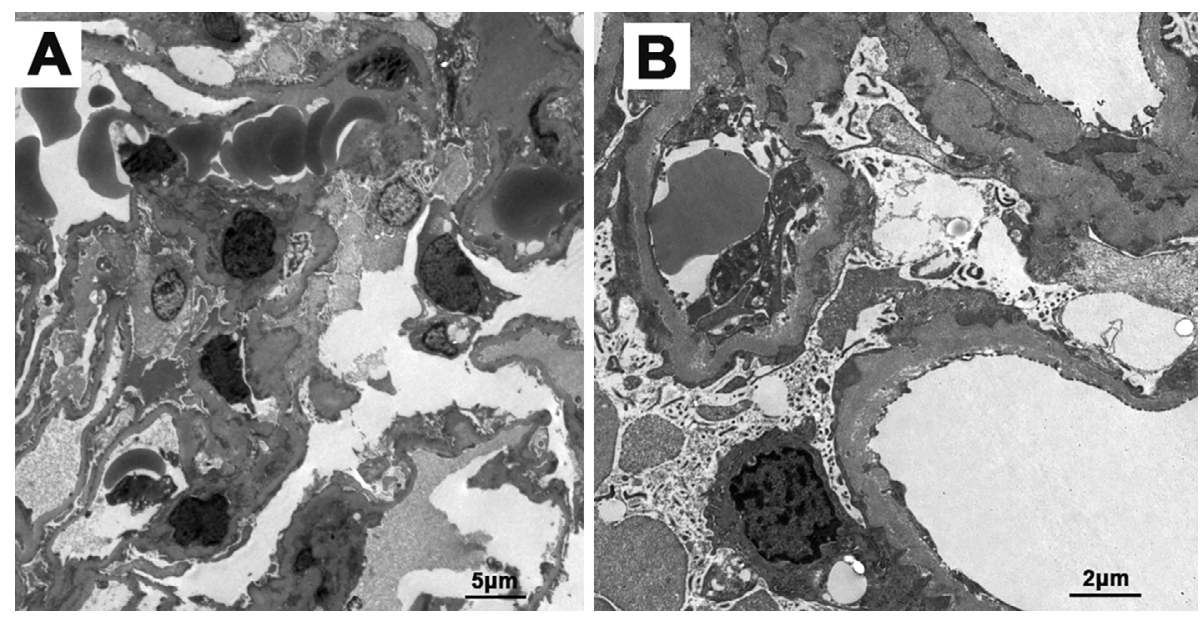

Figure 2. Electron microscopy image showing granular electron-dense deposits and extensive foot process effacement of the podocyte (original magnification $\times 5,000$ ).

pus erythematosus (SLE) and the most important secondary form of $\mathrm{MN}$, was first suspected in our case due to his persistent proteinuria, which is the most frequent abnormality in $\mathrm{LN}$ patients. However, long-term surveillance with various examinations yielded no typical extra-renal signs in the skin, joints or blood, and immunofluorescent microscopy observed no characteristic "full-house" pattern with positive staining for IgG, IgA, IgM, C3 or C1q in SLE, all of which allowed for the exclusion of LN in our case.

Of note, only two cases of IMN with solitary polyclonal IgA deposition have been described previously, namely by Kobayashi et al. (7) in 2015 and by Sawamura et al. (8) in 2019. Table summarizes the clinicopathological findings in the previously reported cases and our present case. These three Asian patients developed NS with a preserved renal function. Anti-nuclear antibodies, cryoglobulin and monoclonal proteins were not detected. Consistent membranous features accompanied by solitary IgA immune deposits were displayed in the current three cases, and positive $\kappa$ - and $\lambda$ light chains along the glomerular capillary walls further confirmed the polyclonal origin of the $\operatorname{IgA}$ immune deposits. With respect to the consistent immune features in three cases, the deficiency of PLA2R and C3 may have profound implications. Previous evidence documented that a positive staining with PLA2R on a renal biopsy was able to identify IMN with $97 \%$ specificity (11), and most patients diagnosed with IMN have IgG4-dominant subepithelial immune complex deposits that colocalize with the PLA2R to podocytes. The deficiency of PLA2R in these three cases thus suggests that other known autoantigens or exogenous mucosal antigens, such as bacterial antigens, may be associated with solitary polyclonal IgA deposition. Furthermore, C3 deposits are another hallmark seen in more than half of IMN patients (6), so we speculate that the shortage in complement deposition may be related to the special mechanism underlying the onset of solitary $\operatorname{IgA}$ deposition, and complement activation may be dispensable during this process.

Notably, low- or medium-dose prednisone therapy was ef- fective for treating NS in the two previously reported cases, while the combined use of medium-dose prednisone and tacrolimus induced remission in our present case. In brief, the quick response to induction therapy suggests a high incidence of remission in this pathological subtype, and the lack of any record of relapse over a long follow-up strengthens our belief in a good prognosis for this unique subtype, despite the fact that the predictable correlation between this pathological subtype with remission rat and recurrence rate remains undetermined. All things considered, we believe these three rare cases call into question the established theories and may help clarify the mechanistic variety involved in the development of MN.

Although the physiological and cellular mechanisms underlying the solitary $\operatorname{IgA}$ deposition in MN remains elusive, there are several variants of glomerular disease with IgA deposits combined with capillary wall abnormalities. The first entity that should be considered in the differential diagnosis is concomitant $\operatorname{IgA}$ nephropathy (IgAN) and $\mathrm{MN}$ (referred to as combined IgAN-MN). According to the study of the largest patient cohort diagnosed with combined IgAN-MN $(\mathrm{n}=73)$, all patients had mesangial IgA-dominant deposits accompanied by obviously increased mesangial cell proliferation (12). $\operatorname{IgAN}$ is characterized by mesangial deposition of $\operatorname{IgA}$, hence this feature is considered as the most evident point to distinguish combined IgAN-MN from MN, and this highly specific histological attribute of IgAN was not noted in the two previously reported cases or our present case. Furthermore, the presence of capillary wall IgG immune deposits was observed in all cases with combined $\operatorname{IgAN}$ MN (12, 13), although these findings were not observed on immunofluorescence in our current case or the two previous cases. Taken together, the characteristic immunofluorescence findings and pathological features of combined IgAN-MN can ensure the early identification of this entity and help avoid missing a diagnosis of a rare case of MN with solitary IgA deposition.

In addition, given the essential role of $\operatorname{IgA}$ in protecting 
Table. Summary of Three Rare Cases of MN with Solitary IgA Deposition.

\begin{tabular}{|c|c|c|c|}
\hline & Reported case 1[7] & Reported case 2[8] & Present case \\
\hline Age (years) & 71 & 60 & 60 \\
\hline Gender & Female & Male & Male \\
\hline Hypertension & $(+)$ & $(-)$ & $(-)$ \\
\hline Edema & $(+)$ & $(+)$ & $(+)$ \\
\hline Complication & None & T2DM & CHD \\
\hline Proteinuria (g/day or g/g creatinine) & 4.8 & 5.1 & 4.8 \\
\hline Microscopic hematuria (>5 RBC/HPF) & $(+)$ & $(-)$ & $(+)$ \\
\hline Serum albumin $(g / d L)$ & 2.1 & 2.4 & 1.8 \\
\hline Serum creatinine (mg/dL) & 0.8 & 0.79 & 0.62 \\
\hline White blood cell (/uL) & ND & 5,900 & 5,560 \\
\hline Hemoglobin $(\mathrm{g} / \mathrm{dL})$ & 10.3 & 14.7 & 13.9 \\
\hline Platelet (/uL) & ND & 191,000 & 226,000 \\
\hline Serum C3 (mg/dL) & 147 & 80 & 127 \\
\hline Serum C4 (mg/dL) & 32 & 12 & 16 \\
\hline Hepatitis B virus antigen & $(-)$ & $(-)$ & $(-)$ \\
\hline Serum antinuclear anti-body & $(-)$ & $(-)$ & $(-)$ \\
\hline Serum cryoglobulin & ND & $(-)$ & $(-)$ \\
\hline Serum IgG (mg/dL) & 1,030 & 572 & 728 \\
\hline Serum $\operatorname{IgA}(\mathrm{mg} / \mathrm{dL})$ & 271 & 345 & 272 \\
\hline Serum IgM (mg/dL) & ND & 93 & 128 \\
\hline \multicolumn{4}{|l|}{ Monoclonal Protein } \\
\hline Serum & $(-)$ & $(-)$ & $(-)$ \\
\hline Urine & $(-)$ & $(-)$ & $(-)$ \\
\hline Treatment (Initial dose) & PSL (25 mg/day) & PSL (10 mg/day) & PSL (30 mg/day)+TAC (2 mg Q12H) \\
\hline Follow-up period (year) & 8 & 3 & 1 \\
\hline Proteinuria ( $\mathrm{g} /$ day or $\mathrm{g} / \mathrm{g}$ creatinine) at follow-up & 2 & 2.1 & $<0.15$ \\
\hline Serum creatinine $(\mathrm{mg} / \mathrm{dL})$ at follow-up & ND & 0.87 & 0.79 \\
\hline \multicolumn{4}{|l|}{ Light microscopy } \\
\hline No. of glomeruli & 12 & 20 & 11 \\
\hline No. of sclerosis & 1 & 2 & 1 \\
\hline GBM thickening & $(+)$ & $(-)$ & $(+)$ \\
\hline Bubbling/spike appearance & $(+)$ & $(-)$ & $(-)$ \\
\hline Mesangial proliferation & $(-)$ & $(-)$ & Mild \\
\hline Interstitial lymphocyte infiltration & ND & Mild & Mild \\
\hline Tubular atrophy & ND & Mild & Mild \\
\hline Interstitial fibrosis & ND & Mild & Mild \\
\hline Vascular alterations & ND & Moderate & Moderate \\
\hline \multicolumn{4}{|l|}{ Immunofluorescence microscopy } \\
\hline $\operatorname{IgG}$ & $(-)$ & $(-)$ & $(-)$ \\
\hline $\operatorname{Ig} \mathrm{A}$ & $(+)$ & $\operatorname{IgA} 1 / \operatorname{IgA} 2(+) /(-)$ & $(+)$ \\
\hline $\operatorname{IgM}$ & $(-)$ & $(-)$ & $(-)$ \\
\hline$\kappa / \lambda$ & $(+) /(+)$ & $(+) /(+)$ & $(+) /(+)$ \\
\hline $\mathrm{C} 3$ & $(-)$ & Trace-positive & $(-)$ \\
\hline $\mathrm{C} 1 \mathrm{q}$ & $(-)$ & $(-)$ & $(+)$ \\
\hline PLA2R & ND & $(-)$ & $(-)$ \\
\hline \multicolumn{4}{|l|}{ Electron microscopy } \\
\hline Subepithelial granular deposits (MN stage) & $(+)(\mathrm{I}$ to II) & $(+)$ (early) & $(+)$ (early) \\
\hline Subendothelial granular deposits & $(-)$ & $(-)$ & $(-)$ \\
\hline Mesangial granular deposits & $(-)$ & $(-)$ & Mild \\
\hline
\end{tabular}

CHD: coronary heart disease, GBM: glomerular basement membrane, HPF: high-power field, MN: membranous nephropathy, ND: not determined, PLA2R: phospholipase A2 receptor, PSL: prednisolone, RBC: red blood cells, T2DM: type 2 diabetes mellitus, TAC: tacrolimus, T2DM: type 2 diabetes mellitus

against infections on mucosal surfaces, such as those exposed to tears, saliva, colostrum, genital, respiratory or gastrointestinal secretions, a hypothesis concerning postinfec- tious glomerular nephritis (PIGN) was proposed. Most cases of PIGN are triggered by nephritogenic strains of group A beta-hemolytic streptococcus, with less common pathogens 
being nonstreptococcal bacteria, viruses, parasites, rickettsiae and fungi. The clinical presentation of PIGN varies from asymptomatic, microscopic hematuria to full-blown acute nephritic syndrome characterized by red to brown urine, proteinuria, edema, hypertension and acute kidney injury. We should also mention that clinical evidence concerning infection was not detected in the skin, respiratory or gastrointestinal systems in our case. Furthermore, classical PIGN always involves C3 and IgG or sole C3 deposition, which is helpful for differentiating PIGN from the condition in our current case based on histological characteristics (14). Another variant that should be differentiated is IgAdominant acute postinfectious glomerulonephritis (APIGN). IgA-dominant APIGN always occurs in association with Staphylococcal infection and manifests as severe acute kidney injury, proteinuria and hematuria, with $\operatorname{IgA}$ being the sole or dominant immunoglobulin on immunofluorescence (15). However, our current case and the other two previously reported patients showed no evidence of concurrent infection, even after long-term surveillance with various examinations. Therefore, the diagnosis of IgA-dominant APIGN could not be established in any of these three patients.

In addition, a review of the literature revealed several unusual case reports of IgA-type monoclonal immunoglobulin deposition disease (MIDD), which is morphologically similar to $\mathrm{MN}$ but contains immunoglobulins derived from single B-cell clones. Miura et al. (16) described a case of solitary $\operatorname{Ig} \mathrm{A} 1-\lambda$ deposition in a granular pattern along the glomerular capillary walls in a patient with chronic hepatitis $\mathrm{C}$ viral infection, and Kitazawa et al. (17) presented another unusual case of MIDD with $\operatorname{IgA} 1-\lambda$ positively stained by immunofluorescence on a renal biopsy with membranous features. Intriguingly, Sethi (18) described a monoclonal IgA-type MN patient with diffuse granular deposition of $\operatorname{IgA}-\kappa$ along the glomerular capillary walls in a renal biopsy, which was deemed monoclonal gammopathy with an undetermined underlying etiology. However, despite the similar membranous features among monoclonal IgA-type $\mathrm{MN}$, IgA-type MIDD and MN with solitary $\operatorname{IgA}$ deposition, the coexistence of $\kappa$ - and $\lambda$-light chains on immunofluorescence not only highlights the polyclonal origin of the $\operatorname{IgA}$ immune deposits in our current observation and the two previous cases but also makes the diagnosis of monoclonal IgA-type MN or IgA-type MIDD unlikely.

In conclusion, IMN associated with solitary polyclonal $\operatorname{Ig} \mathrm{A}$ deposition is an extremely rare entity. Our current case and the two previously reported cases highlight the unique pathological nature of this entity, and future studies of similar cases are needed to outline the potential utility of these findings in guiding personalized therapy.

The authors state that they have no Conflict of Interest (COI).

\section{Acknowledgement}

We wish to thank our patient for allowing us to publish his case.

\section{Consent for publication}

Written informed consent was obtained from the patient for the publication of this case report, including clinical information and related images. The consent form has been stored by the corresponding author and may be obtained on request from the editor of this journal.

\section{Funding}

This study was supported by International Postdoctoral Exchange Fellowship Program of China (Talent-Introduction Program, YJ20200210), and the National Natural Science Foundation of China (82100747). The funder had no role in the design of the study or in the collection, analysis or interpretation of the data.

\section{Authors' contributions}

B.L. and H.L.H. participated in the treatment as primary physicians, acquired the data and drafted the initial manuscript. S.C. Y. performed the histopathological examinations and obtained the diagnosis. X.H.C. supervised and designed the study. X.W., W. C., Z.J.L. and X.H.C. revised the manuscript. All authors have read and approved the final version of this manuscript.

\section{References}

1. Radice A, Trezzi B, Maggiore U, et al. Clinical usefulness of autoantibodies to M-type phospholipase A2 receptor (PLA2R) for monitoring disease activity in idiopathic membranous nephropathy (IMN). Autoimmun Rev 15: 146-154, 2016.

2. Roccatello D, Sciascia S, Di Simone D, et al. New insights into immune mechanisms underlying response to Rituximab in patients with membranous nephropathy: A prospective study and a review of the literature. Autoimmun Rev 15: 529-538, 2016.

3. Huang CC, Lehman A, Albawardi A, et al. IgG subclass staining in renal biopsies with membranous glomerulonephritis indicates subclass switch during disease progression. Mod Pathol 26: 799805, 2013.

4. Davies DR, Tighe JR, Wing AJ, Jones NF. Immunoglobulin deposition in membranous glomerulonephritis: immunofluorescence and immuno-electron microscopy findings. Histopathology 1: 3952, 1977.

5. Qu Z, Liu G, Li J, et al. Absence of glomerular IgG4 deposition in patients with membranous nephropathy may indicate malignancy. Nephrol Dial Transplant 27: 1931-1937, 2012.

6. Ma H, Sandor DG, Beck LH Jr. The role of complement in membranous nephropathy. Semin Nephrol 33: 531-542, 2013.

7. Kobayashi M, Usui J, Sakai K, et al. Membranous nephropathy with solitary immunoglobulin A deposition. Intern Med 54: 10811084, 2015.

8. Sawamura M, Komatsuda A, Kaga H, et al. Membranous nephropathy with solitary polyclonal IgA deposition: A case report and literature review. Clin Nephrol Case Stud 7: 60-65, 2019.

9. Improving Global Outcomes (KDIGO) Glomerulonephritis Work Group. KDIGO Clinical Practice Guideline for Glomerulonephritis. Kidney Inter Suppl 2: 139-274, 2012.

10. von Haxthausen F, Reinhard L, Pinnschmidt HO, et al. AntigenSpecific IgG Subclasses in Primary and Malignancy-Associated Membranous Nephropathy. Front Immunol 9: 3035, 2018.

11. Dai H, Zhang H, He Y. Diagnostic accuracy of PLA2R autoantibodies and glomerular staining for the differentiation of idiopathic 
and secondary membranous nephropathy: an updated metaanalysis. Scientific reports 5: 8803, 2015.

12. Chen X, Chen Y, Shi K, et al. Comparison of prognostic, clinical, and renal histopathological characteristics of overlapping idiopathic membranous nephropathy and IgA nephropathy versus idiopathic membranous nephropathy. Scientific reports 7: 11468, 2017.

13. Hu R, Xing G, Wu H, Zhang Z. Clinicopathological features of idiopathic membranous nephropathy combined with IgA nephropathy: a retrospective analysis of 9 cases. Diagn Pathol 11: 86, 2016.

14. Nasr SH, Valeri AM, Cornell LD, et al. Renal monoclonal immunoglobulin deposition disease: a report of 64 patients from a single institution. Clin J Am Soc Nephrol 7: 231-239, 2012.

15. Nasr SH, D'Agati VD. IgA-dominant postinfectious glomerulonephritis: a new twist on an old disease. Nephron Clin Pract 119: c18-c25; discussion c26, 2011.
16. Miura N, Uemura Y, Suzuki N, et al. An IgA1-lambda-type monoclonal immunoglobulin deposition disease associated with membranous features in a patient with chronic hepatitis $\mathrm{C}$ viral infection and rectal cancer. Clin Exp Nephrol 14: 90-93, 2010.

17. Kitazawa A, Koda R, Yoshino A, Ueda Y, Takeda T. An IgA1lambda-type monoclonal immunoglobulin deposition disease associated with membranous features in a patient with IgG4-related kidney disease: a case report. BMC Nephrol 19: 330, 2018.

18. Sethi S. Membranous glomerulonephritis with monoclonal immune deposits and crescents. Nephrol Dial Transplant 20: 17681769, 2005.

The Internal Medicine is an Open Access journal distributed under the Creative Commons Attribution-NonCommercial-NoDerivatives 4.0 International License. To view the details of this license, please visit (https://creativecommons.org/licenses/ by-nc-nd/4.0/).

\section{(C) The Japanese Society of Internal Medicine Intern Med Advance Publication}

